\title{
PAPR Reduction Using Interleaver-Assisted PTS Algorithm for OFDM Wireless System
}

\author{
A. N. Jabir \\ Dijlah University College, Baghdad, Iraq, \\ amjad.najim@duc.edu.iq
}

\begin{abstract}
This paper presents a new approach to tackle the problem of high Peak to Average Power Ratio (PAPR) in Orthogonal Frequency Division Multiplexing (OFDM) systems. The contents of each OFDM symbol are randomly permuted before being applied to the Partial Transmit Sequence (PTS) algorithm. This results in a greater chance of finding a candidate symbol within the constraints of the power amplifier at the transmitter side compared to the original PTS approach. Computer Simulations show a gain of least $1.5 \mathrm{~dB}$ at the probability value of $10^{-4}$ for the tested systems.
\end{abstract}

Key words: OFDM, PAPR, PTS, Interleaver.

\section{INTRODUCTION}

Multicarrier OFDM systems made it possible to have such a variety of today's data transmission and mobile services which all require high data rates and sophisticated means to tackle impairments caused by communication channels and for the most efficient use of their bandwidth. OFDM has been adopted as part of the IEEE 802.11a and 802.11g standards for high bit rate data transmission over wireless LANs as well as other standards such as digital audio broadcasting (DAB), digital video broadcasting (DVB), the European HIPERLAN/2 [1], [2], [3]. For a single carrier digital transmission system with a modulation scheme such as $M$-QAM or $M$-PSK, a symbol duration of $T_{s}$, a channel of bandwidth $B$ and a delay spread of $\tau$, the following condition has to be satisfied in order to have a reception of no inter symbol interference (ISI) [4]:

$$
\tau \ll T_{s}
$$

which can limit the highest possible data rate. In multi carrier OFDM systems, $B$ is divided into $N$ sub bands among $N$ orthogonal subcarriers. The main serial input symbol stream is first partitioned into $N$ sub streams the symbol rate on each is $N T_{s}$ instead of $T_{s}$. As such, every $T=N T_{s}$ an OFDM symbol consisting of $N$ symbols is transmitted through the channel after being up converted by the final carrier frequency $f_{c}$. The subcarriers frequencies are $k \Delta f, k=0, \cdots, N-1$ where $\Delta f=\frac{1}{T}$ in order satisfy the orthogonality condition on the sub carriers which facilitates fluctuations in its time domain signal which can draw the power amplifier into saturation leading to the harmful effects of non linear distortion and spectral spreading [5]. The PAPR problem in OFDM systems has been extensively studied and is still an active research topic [1, 6, 7, 8, 9, and 10]. The proposed solutions offer different grades of tradeoff between computational complexity and performance in terms of PAPR, Bit Error Rate (BER) and data rate loss due to the transmission of side information necessary for the demodulation operation at the receiver side.

\section{PAPR IN OFDM SYSTEMS}

The transmitter side of an OFDM system is shown in Figure (1). The continuous time baseband signal with $N$ orthogonal subcarriers is given by [11, eq. 7.1, page 202]:

$$
x(t)=\frac{1}{\sqrt{N}} \sum_{k=0}^{N-1} X[k] e^{j 2 \pi k \Delta f t}, 0 \leq t \leq T
$$

$j=\sqrt{-1}, T$ is the OFDM symbol time which is equal to $N T_{S}$ with $T_{s}$ as the symbol time of the incoming serial modulated symbols $X[k]^{\prime}$ 's, $k \Delta f$ is the frequency of the $k$ th subcarrier and $\Delta f=\frac{1}{T}$. The signal $x(t)$ is a vector sum of $N$ signals for each value of $t$ which may lead to large amplitudes if added constructively. The up converted signal by carrier $f_{c}$ is just the real part of $x(t) e^{j 2 \pi f_{c} t}$.

Setting $t=n \Delta t$ with $\Delta t=\frac{T}{N}$ in (1) we get the discrete time domain (DT) representation of the signal $x(t)$ as:

$$
x[n]=\frac{1}{\sqrt{N}} \sum_{k=0}^{N-1} X[k] e^{j 2 \pi k \frac{n}{N}}, 0 \leq n \leq N-1
$$

It is evident from (2) that $x[n]$ is just the IDFT of the vector $X[k]$.

The PAPR is defined as:

$$
P A P R=\frac{\max _{0 \leq t \leq T}|x(t)|^{2}}{E\left\{|x(t)|^{2}\right\}}
$$




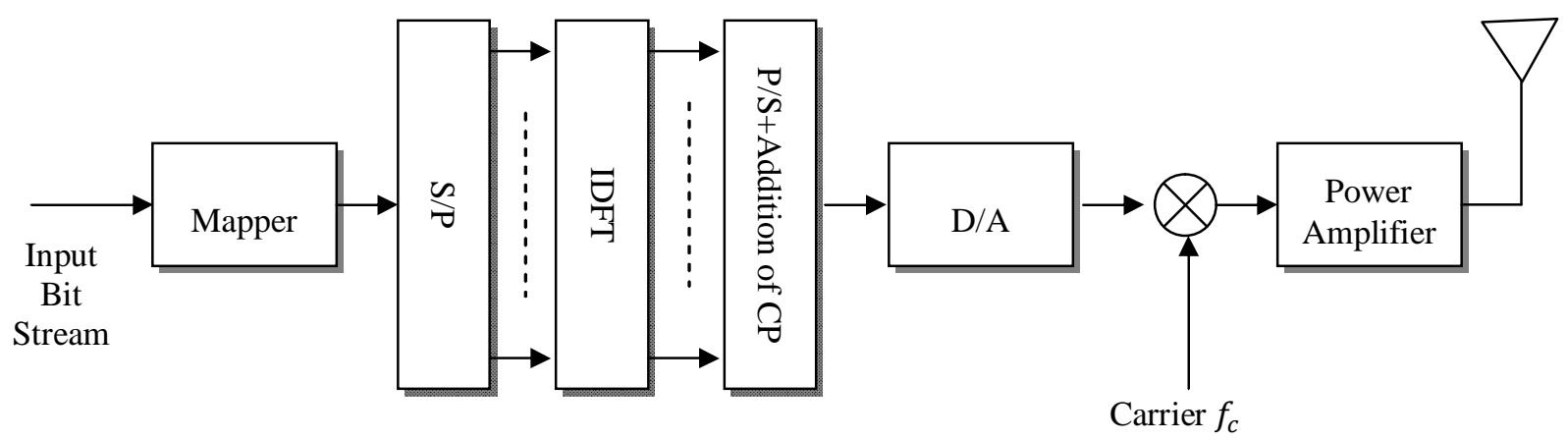

Figure 1: Transmitter side of an OFDM system

or, in the discrete time (DT) domain:

$$
P A P R=\frac{\max _{0 \leq n \leq N-1}|x[n]|^{2}}{E\left\{|x[n]|^{2}\right\}}
$$

where $E$ is the expectation operator.

In order not to miss the peak values of $x[n]$ in the DT domain for the calculation of PAPR we have to do over sampling using the sampling frequency of $f_{S}=L \frac{N}{T}$ instead of $\frac{N}{T}$ where $L$ is called the oversampling factor. With oversampling, $x[n]$ is therefore expressed as $[12$, eq. 2.5 , page 25]:

$$
x[n]=\frac{1}{\sqrt{N}} \sum_{k=0}^{N-1} X[k] e^{j 2 \pi k \frac{n}{L N}}, 0 \leq n \leq L N-1
$$

where $x[n]$ is assumed to mean $x\left[n \frac{T}{L N}\right]$ and the sampling time is $\frac{T}{L N}\left(=\frac{1}{f_{S}}\right)$.

Oversampling can also done using DFT properties for real time signals and zero padding in the frequency domain as in [13] and [14].

\section{STATISTICAL PROPERTIES OF THE PAPR}

Statistical behaviour of the PAPR is usually described in terms of complementary cumulative distribution function (CCDF) which describes the probability that the OFDM signal envelope exceeds a certain threshold, $\delta$,

$$
\operatorname{CCDF}(\delta)=\operatorname{Prob}(P A P R>\delta)
$$

If the real and imaginary parts of $x[n]$ are assumed to be statistically independent Gaussian random variables then from central limit theory the envelope of the OFDM signal will follow a Rayleigh distribution and the energy distribution becomes an exponential, or equivalently, a central chi-square distribution with two degrees of freedom with a CCDF given by[15][16]:

$$
\operatorname{CCDF}(\delta)=1-\left(1-e^{-\delta}\right)
$$

If the $N$ samples of $x[n]$ are assumed to be mutually independent, then:

$$
\operatorname{CCDF}(\delta)=1-\left(1-e^{-\delta}\right)^{N}
$$

Since $x[n]$ is discrete this does not necessarily mean that the peak value of the continuous time signal $x(t)$ has been considered.

Oversampling can help in this regard although that this could violate the assumption of independence between the signal samples. Taking oversampling into consideration, the CCDF can be expressed as [1]:

$$
C D F(\delta)=1-\left(1-e^{-\delta}\right)^{\alpha N}
$$

where $\alpha$ is the oversampling factor.

Figure (2) shows the above relationship of (7) for the values of $\delta$ from 4 to $13 \mathrm{~dB}$ with different values of number of subcarriers,

\section{THE PROPOSED APPROACH}

The proposed method is shown in Figure (3). It differs from the Partial Transmit Sequence (PTS) of [17] by doing a random permutation on the symbol sequence out of the S/P converter before pushing it to the $\frac{N}{M}$ IFFT blocks where $M$ is the block size. It is thought that with the use of the interleaver it would be more likely to get an OFDM symbol candidate with a lower PAPR. 


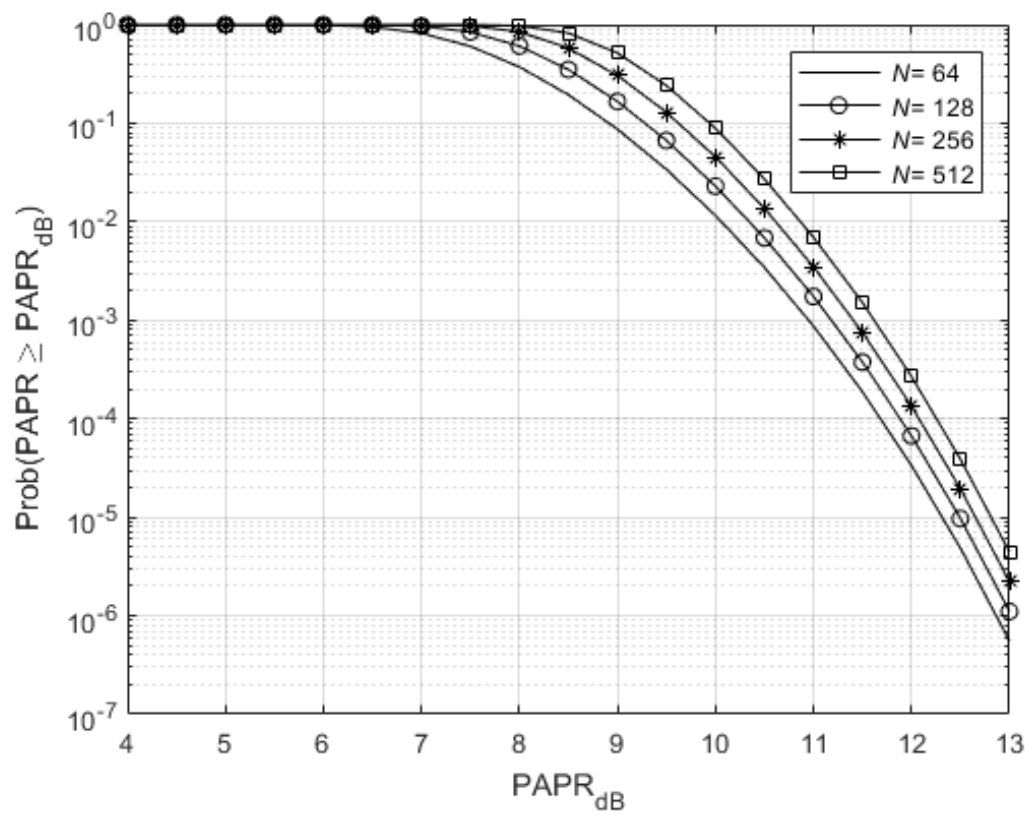

Figure 2: Theoretical CCDF of PAPR for different values of sub carriers $N$ (no oversampling)

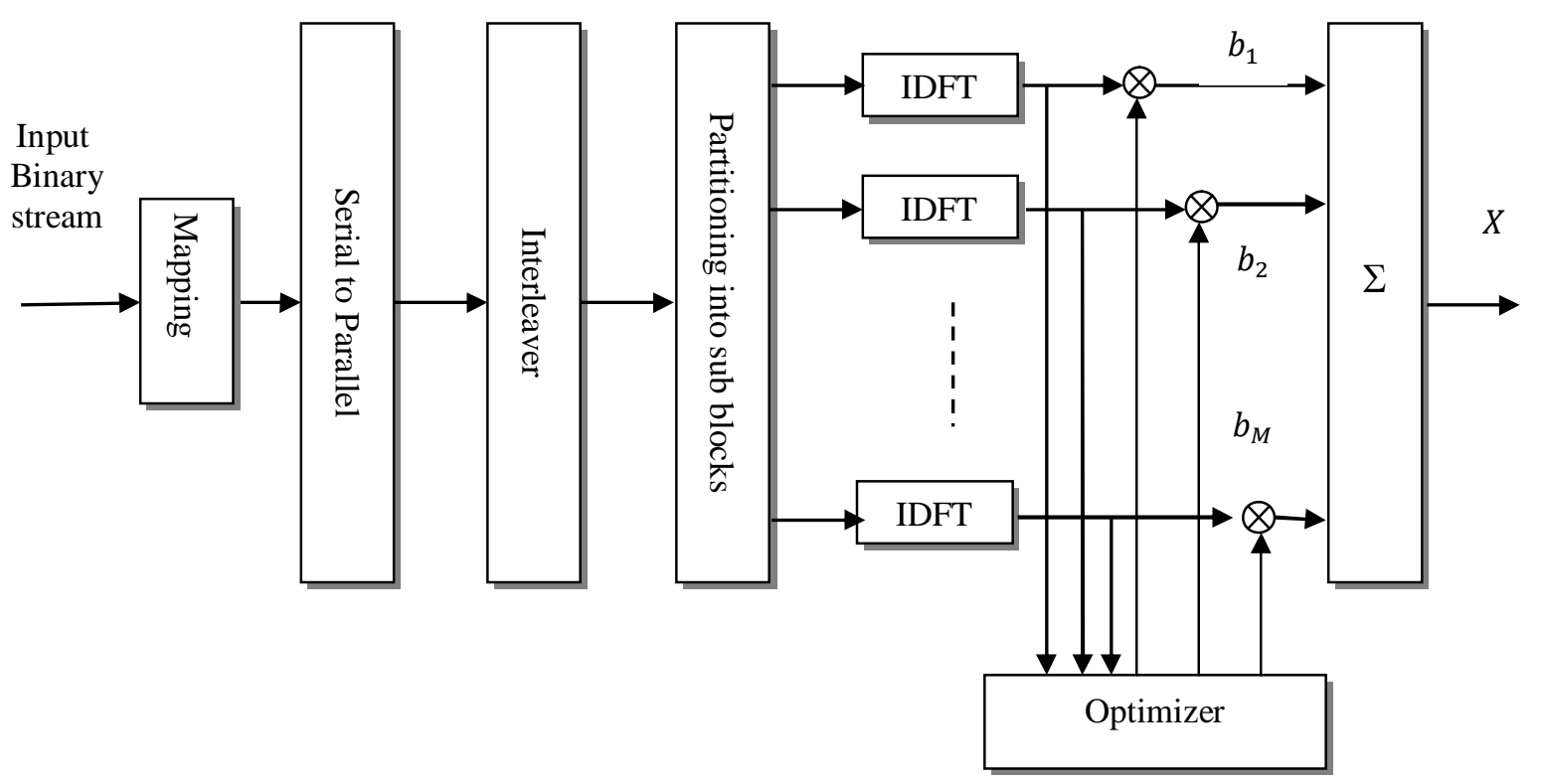

Figure 3 : The proposed method

\section{RESULTS AND DISCUSSION}

Performance of the proposed method was tested using computer simulations with Matlab 2017 (Licensed Student Edition) using QPSK and different values of number of sub carriers $N$ and the oversampling factor $L$ for the range of PAPR values from 4 to $13 \mathrm{~dB}$. In order to get reasonably accurate results a very large number of realizations was selected of $10^{6}$. Another possible approach is to make whatever number of realizations it takes to reach a certain probability value. A comparison was made with the PTS method.

Figures (4) shows the result for the case of $N=128$, QPSK, $L=1$ while Figure (5) is for the case of $N=256$, QPSK, $L=4$. It is evident from these figures that a gain of at least $1.5 \mathrm{~dB}$ has been achieved at the probability of $10^{-4}$. 


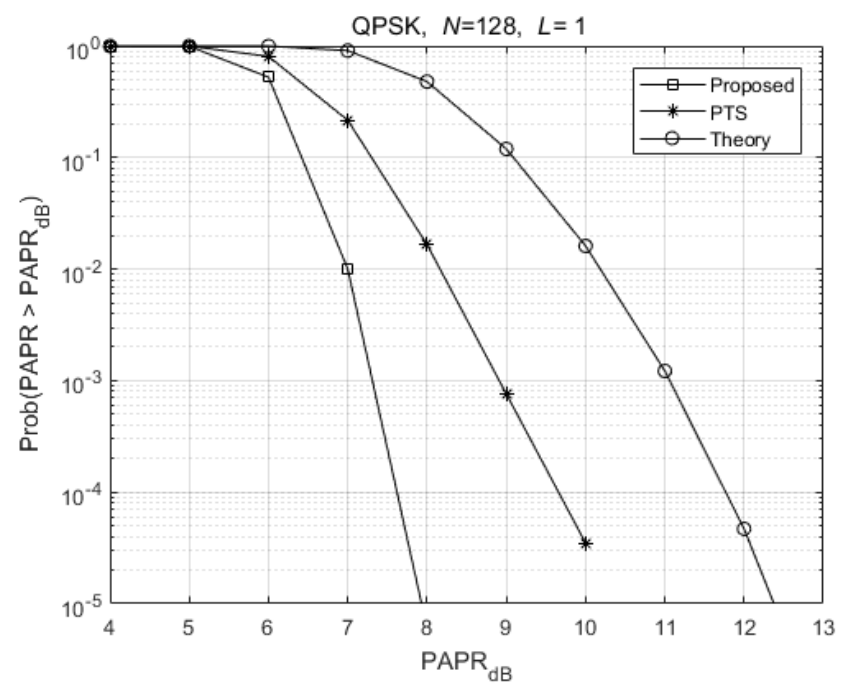

Figure (4): Proposed method performance with QPSK, $N=128, L=1$.

This remarkable improvement comes at the cost of a slight increase in complexity (due to doing the permutations) which may be justified for certain applications.

The computational complexity of the original PTS can be found as follows:

The complexity of the IFFT block using a sequence of length $N$ is $\frac{N}{2} \log _{2}(N)$ complex multiplications and $N \log _{2}(N)$ complex additions [18] or equivalently $2 N \log _{2}(N)$ real multiplications and $3 N \log _{2}(N)$ real additions. The complexity of the PTS is shown in Table (1) per decision. The complexity of the proposed method is slightly higher by a factor that depends on the allowed number of permutations of the interleaver. Table 1 summarizes the computational complexity findings of the PTS algorithm.

\section{CONCLUSION}

A method has been proposed for the reduction of PAPR in OFDM systems that offers a gain of at least $1.5 \mathrm{~dB}$ at the probability value of $10^{-4}$ over the PTS known approach with slight increase in complexity which might be affordable in some systems. As with the original PTS, side information has to be sent to the receiver side

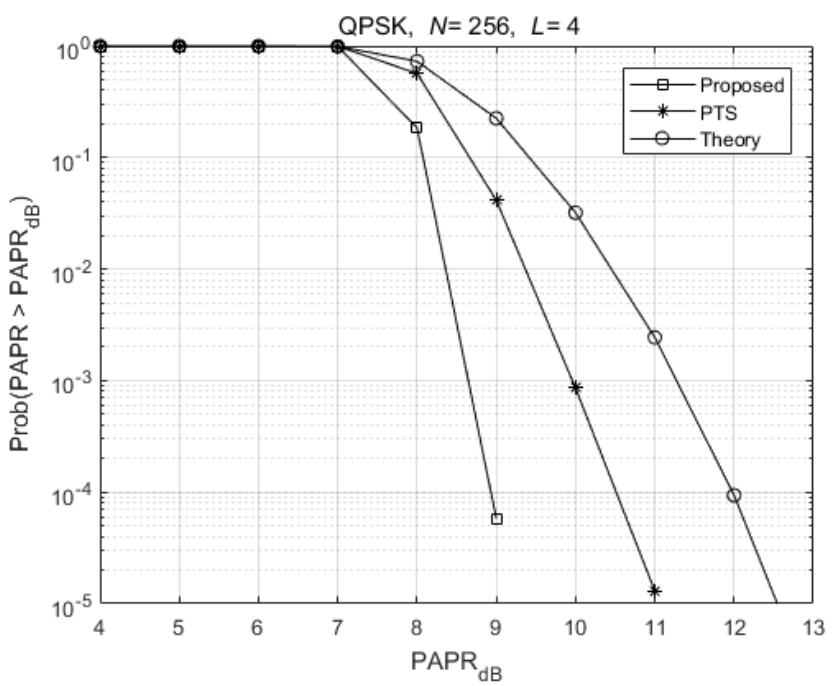

Figure (5): Proposed method performance with QPSK, $N=256, L=4$.

Table 1: Computational Complexity of the PTS Algorithm

\begin{tabular}{|c|c|c|}
\hline Operation & $\begin{array}{c}\text { Number of Real } \\
\text { multiplications }\end{array}$ & $\begin{array}{c}\text { Number of Real } \\
\text { additions }\end{array}$ \\
\hline IFFT & $B\left[2 N L \log _{2}(L N)\right]$ & $B\left[3 N L \log _{2}(L N)\right]$ \\
\hline $\begin{array}{c}\text { Complex } \\
\text { weighting }\end{array}$ & $4 B L N$ & $2 B L N$ \\
\hline $\begin{array}{c}\text { Finding } \\
\text { PAPR }\end{array}$ & $4 B L N$ & $2 B L N+B(L N-1)$ \\
\hline $\begin{array}{c}\text { Selecting } \\
\text { lowest PAPR }\end{array}$ & $B-1$ \\
\hline \multicolumn{2}{|c|}{$N:$ number of sub carriers, $L:$ oversampling factor, } \\
$M:$ Block size, $B=\frac{N}{M}:$ Number of Blocks
\end{tabular}




\section{REFERENCES}

1. Yasir Rahmatallah and Seshadri Mohan, Peak-ToAverage Power Ratio Reduction in OFDM Systems: A Survey and Taxonomy, IEEE Communications Surveys \& Tutorials, Vol. 15, no. 4, pp. 1567-1592, fourth quarter 2013. https://doi.org/10.1109/SURV.2013.021313.00164

2. R. V. Nee, G. Awater, M. Morikura, H. Takanashi, M. Webster, and K. W. Halford, New high-rate wireless LAN standards, IEEE Communication Magazine, Vol. 37, no. 12, pp. 82-88, December 1999.

3. Koffman and V. Roman, Broadband wireless access solution based on OFDM access in IEEE 802.16, IEEE Communication Magazine, Vol. 40, no. 4, pp. 96-103, April 2002. https://doi.org/10.1109/35.995857

4. H. Schulze and C. Luders, Theory and Applications of OFDM and CDM, Wiley, 2005, ch. 4 , p. 145.

https://doi.org/10.1002/0470017406

5. H. Ochiai and H. Imai, On the distribution of the peak-to-average power ratio in OFDM signals, IEEE Transactions on Communications, Vol. 49, no. 2, pp. 282-289, February 2001.

6. X. Li and L. J. C. Jr., Effect of clipping and filtering on the performance of OFDM, IEEE Communication Letters, Vol. 2, no. 5, pp. 131-133, May 1998.

https://doi.org/10.1109/4234.673657

7. S. H. Han and J. H. Lee, An overview of peak-toaverage power ratio reduction techniques for multicarrier transmission, IEEE Wireless Communications, Vol. 12, no. 2, pp. 56-65, Apr. 2005.

8. T. Jiang and $\mathrm{Y}$. $\mathrm{Wu}$, An Overview: peak-toaverage power ratio reduction techniques for OFDM signals, IEEE Transactions on Broadcasting, Vol. 54, no. 2, pp. 257-268, June 2008.

9. Sohn, I. and Chul, S., Neural network based simplified clipping and filtering technique for PAPR reduction of OFDM signals, IEEE Communication Letters, Vol. 19, pp. 1438-1441, 2015.

10. M. Kim, W. Lee and D. Cho, A Novel PAPR Reduction Scheme for OFDM System Based on Deep Learning, IEEE Communications Letters, Vol. 22, no. 3, pp. 510-513, March 2018.

11. Man-On Pun, Michele Morelli and C-C Jay Kuo, Multi-Carrier Techniques for Broadband Wireless Communications: A Signal Processing Perspective, Imperial College Press, 2007.

12. Emad S. Hassan, Multi-Carrier Communication Systems with Examples in MATLAB, A New Perspective, CRC Press, 2016, ch. 2, p. 25. https://doi.org/10.1201/b19273
13. S. C. Thompson, A. U. Ahmed, J. G. Proakis, J. R. Zeidler and M. J. Geile, Constant Envelope OFDM, in IEEE Transactions on Communications, Vol. 56, no. 8, pp. 1300-1312, August 2008. https://doi.org/10.1109/TCOMM.2008.070043

14. I. Sohn, A Low Complexity PAPR Reduction Scheme for OFDM Systems via Neural Networks, in IEEE Communications Letters, Vol. 18, no. 2, pp. 225-228, February 2014.

15. R. V. Nee and A. D. Wild, Reducing the peak-toaverage power ratio of OFDM, in Proc. IEEE Vehicular Technology Conference (VTC), Vol. 3, New York, USA, 1998, pp. 2072-2076.

16. T. Jiang and $\mathrm{Y}$. Wu, An Overview: peak-toaverage power ratio reduction techniques for OFDM signals, IEEE Transactions on Broadcasting, Vol. 54, no. 2, pp. 257-268, June 2008. https://doi.org/10.1109/TBC.2008.915770

17. S. H. M“uller and J. B. Huber, OFDM with reduced peak-to average power ratio by optimum combination of partial transmit sequences, IEEE Electronic Letters, Vol. 33, no. 5, pp. 368-369, 1997. https://doi.org/10.1049/el:19970266

18. B. P. Lathi, and Roger A. Green, Essentials of Digital Signal Processing, Cambridge University Press, 2014. 